\title{
LIDERANÇA ORGANIZACIONAL: UMA REVISÃO INTEGRATIVA BRASILEIRA
}

THE ORGANIZATIONAL LEADERSHIP: A BRAZILIAN INTEGRATIVE REVIEW

Recebido em 13.09.2019 Aprovado em 06.03.2020

Avaliado pelo sistema double blind review

DOI: https://doi.org/10.12712/rpca.v14i1.36370

\section{Caio Pedrinho da Silva}

caio_pedrini@hotmail.com

Programa de Pós-Graduação em Administração/Universidade Federal do Paraná - Curitiba/Paraná, Brasil

ORCID: https://orcid.org/0000-0002-4214-8527

\section{Marco Antonio Catussi Paschoalotto}

marcocatussi@unoeste.br

Departamento de Administração/Universidade do Oeste Paulista - Presidente Prudente/São Paulo, Brasil

ORCID: https://orcid.org/0000-0003-2276-8531

\section{Gustavo Yuho Endo}

gustavo@unoeste.br

Departamento de Administração/Universidade do Oeste Paulista - Presidente Prudente/São Paulo, Brasil

ORCID: https://orcid.org/0000-0003-2737-6596

\section{Resumo}

O presente artigo analisou de forma integrativa e sistêmica os trabalhos de liderança publicados em periódicos nacionais de 2010 a 2018 por meio da plataforma CAPES, onde foram filtrados os estudos de liderança com strings de busca e critérios de restrição em 3 etapas, gerando uma amostra final de 29 artigos. Os resultados apontaram que os assuntos mais abordados dentro da temática são a respeito dos estilos de liderança, com foco na transformacional e transacional; e temas como coaching e liderança feminina apresentaram poucos artigos. Ao final, esse estudo demonstrou uma agenda de pesquisa a ser seguida.

Palavras-chave: Liderança. Revisão Sistemática Integrativa. Organização. Estilos de liderança. Gestão de Pessoas.

\begin{abstract}
We have analyzed in an integrative and systemic form, leadership papers published in Brazilian journals from 2010 to 2018 through the CAPES platform, which with search strings and 3-step restriction criteria were filtered, generating a final sample of 29 articles. The results have pointed out the subjects approached from the theme are the respect of leadership styles, focusing on transformational and transactional; and topics such as coaching and female leadership featured few articles. In the end, we have demonstrated a research agenda to be followed.
\end{abstract}

Keywords: Leadership. Systematic Integrative Review. Organization. Leadership styles. Human Resources Management. 


\section{Introdução}

Ao longo dos tempos o conceito de liderança foi se alterando devido as recorrentes mudanças e disseminações de novas ideias aplicadas as organizações. O que antes era sinônimo de chefe ou gestor se modificou para a busca do desenvolvimento organizacional e ramificou em diversos conceitos diferentes pelos pesquisadores (BERGAMINI, 2009; YAMAFUKO; SILVA, 2015).

De acordo com Behnke (2014), a liderança consiste na técnica de influenciar os outros em decorrência do seu poder e capacidade, por meio do diálogo, dos exemplos e das atitudes, alcançando os objetivos pré-estabelecidos e moldando a equipe de acordo com os seus conceitos. Já para Arruda, Chrisóstomo e Rios (2010), a liderança é o processo de se adaptar as mudanças e fazer com que os liderados também se adaptem a isso, descobrindo as suas motivações, as encorajando e as transformando de modo criativo, comunicativo e flexível.

Yamafuko e Silva (2015) exortam que ainda não há uma definição exata de liderança entre os pesquisadores, mas que se trata de um indivíduo que serve aos seus liderados, conhecendo o perfil de cada um a fim de desenvolver suas competências e habilidades. Como complemento, Bertolla et al. (2014) aponta que é necessário que um gestor se transforme em um líder e adentre os assuntos pessoais dos colaboradores, pois eles afetam na produtividade, sendo a influência do gestor/líder sobre a equipe de extrema importância para os resultados da organização.

Dessa forma, um líder pode agir de diversas formas dentro da organização e com diferentes atitudes de acordo com as suas características, da empresa e da sua equipe. Assim, o papel do líder é fundamental, quando posto em prática de forma eficiente e eficaz para o bom sucesso dos objetivos traçados (WOLFF; CABRAL; LOURENÇO, 2013).

Para Ivan e Terra (2017), o líder é vital na dinâmica organizacional ao ser o instrumento de acompanhamento das mutações da sociedade, de forma a atuar de forma intra e inter organizacional. Para isso o líder necessita ser carismático, conhecer sobre gestão de equipes, analisar os estilos de liderança, ser motivador, preparado para mudanças, ser estratégico e multifuncional (AARONS et al., 2016; ARRUDA; CHRISÓSTOMO; RIOS, 2010).

Portanto, a conduta dos líderes influencia na conduta de seus liderados, que consecutivamente procede no alcance dos resultados almejados de forma positiva ou negativa, ao passo de a liderança estar diretamente ligada a motivação dos colaboradores e satisfação dos desejos da organização (CARVALHO; PALMEIRA; MARIANO, 2012; EPITROPAKI et al., 2017). Botelho e Krom (2010) também ressaltam que nenhum estilo de liderança deve ser desprezado, pois é necessário se adaptar a diferentes situações e ambientes, e suprir não apenas os objetivos organizacionais, mas também os objetivos pessoais dos liderados.

Porém, também é necessário cuidado ao lidar com os diversos estilos de liderança empregados pelo líder, pois eles também podem influenciar negativamente os seus liderados. Arnold et al. (2015) exorta que os estilos de liderança estão positivamente correlacionados com a regulação emocional e o esgotamento (Burnout) dos colaboradores.

Dessa forma, os meios de liderança também trouxeram novos métodos para as organizações, entre eles o coaching, que visa o desenvolvimento de competências e habilidades do coachee (colaborador) para se aprimorar em meio ao ambiente de trabalho, a fim de atingir um determinado objetivo (BERG; KARLSEN, 2016; NORO; STÜKER; OLIVEIRA, 2015). Assim como o coaching, estas mudanças na sociedade também vêm incentivando a abordagem e estudos de novas temáticas, como a liderança feminina (ROCHA-COUTINHO; COUTINHO, 2011; SIDANI; KONRAD; KARAM, 2015). 
Outro fator interessante que está relacionado com a liderança é a ética, sendo que a postura transmita pela liderança pode ser um aspecto de influência na representação dos liderados, estando positivamente relacionada com a percepção de justiça e o comportamento ético do colaborador (BEDI; ALPASLAN; GREEN, 2016).

Nesse contexto, é possível notar que os pesquisadores da área de liderança exploraram a forma como os líderes podem ajudar as equipes por meio de uma variedade de atividades (MORGESON; DERUE; KARAM, 2010), como: o coaching (BERG; KARLSEN, 2016; WAGEMAN, 2001), o papel dos líderes na promoção da aprendizagem (WAGEMAN, 2001); como os líderes gerenciam os ocorridos na organização (MORGESON, 2005; MORGESON; DERUE, 2006); o papel dos líderes na organização (AARONS et al., 2016; DRUSKAT; WHEELER, 2003); como as teorias tradicionais de liderança e as teorias contemporâneas de liderança operam nas organizações (ARNOLD et al., 2015; CHEN et al., 2007; EISENBEISS, VAN KNIPPENBERG; BOERNER, 2008; SCHAUBROECK; LAM; CHA, 2007; YAHAYA; EBRAHIM, 2016); e como os papéis de liderança são compartilhados nas equipes (CARSON; TESLUK; MARRONE, 2007; D'INNOCENZO; MATHIEU; KUKENBERGER, 2014; HILLER; DAY; VANCE, 2006; PEARCE; SIMS, 2002).

Todas essas variações nos estudos relacionados à liderança levam a um desejo de conhecer o caminho que os estudos brasileiros tomaram nos últimos anos na área acadêmica. Além disso, conhecer a trajetória brasileira acadêmica da liderança abre espaço para construção de uma agenda de pesquisas organizacionais (FONSECA; PORTO; BORGES-ANDRADE, 2015). Com essa perspectiva, a problemática a ser respondida em nosso estudo é: Como as temáticas de lideranças estão sendo abordadas em artigos acadêmicos publicados em revistas nacionais no período de 2010 a 2018 ?

Para solucionar a problemática, o objetivo geral desse trabalho é analisar de forma integrativa e sistêmica os trabalhos de liderança publicados em periódicos nacionais na plataforma da CAPES de 2010 a 2018. Como objetivos específicos: ampliar o embasamento teórico sobre a temática de liderança; analisar quantitativamente os estudos nacionais de liderança publicados de 2010 a 2018 na plataforma CAPES de forma sistemática e integrativa; comparar qualitativamente os estudos analisados a partir das temáticas descritas e; propor uma agenda de pesquisa nacional para a área de liderança nos próximos anos.

O presente estudo justifica-se ao: colaborar com os estudos científicos sobre a liderança nas organizações por meio de uma revisão sistemática integrativa; ampliar o olhar da comunidade acadêmica sobre a liderança; aprofundar a compreensão de aspectos específicos de liderança; direcionar para futuras pesquisas com potencial de impacto na área de liderança e; correlacionar os caminhos tomados pelos estudos sobre a liderança desde o ano de 2010.

Para melhor compreensão do trabalho, foi realizada a seguinte divisão: nessa primeira seção apresenta-se a contextualização acerca da temática de liderança, juntamente com os objetivos propostos e a justificativa da pesquisa; na segunda seção são apresentados os métodos que foram utilizados para alcançar o objetivo proposto e os passos utilizados nessa pesquisa possibilitando sua replicação; na terceira seção são apresentados os resultados quantitativos e qualitativos juntamente com as discussões a luz da teoria apresentada e; por fim, na última seção são apresentadas as considerações finais do estudo e as sugestões de trabalhos futuros sobre a temática.

\section{Procedimentos Metodológicos}

O referente estudo conta com uma abordagem qualitativa e quantitativa, ao compreender com mais precisão a temática investigada e descrever os dados de forma numérica e analítica (MASCARENHAS, 2012). A coleta de dados foi realizada também de forma bibliográfica, que consiste no procedimento de buscar por soluções e alcançar os objetivos do estudo (LIMA; MIOTO, 2007), com foco na temática de 
liderança, utilizando fontes primárias. Foram coletados estudos desenvolvidos entre os anos de 2010 a 2018 na plataforma CAPES.

Para o procedimento de coleta e análise de dados foi utilizada da revisão sistemática integrativa. A revisão sistemática é a análise sintética de estudos desenvolvidos previamente com metas, ferramentas e passos definidos de forma clara e com possível reprodução (GREENHALGH, 1997, p. 672), enquanto a forma integrativa reúne várias pesquisas publicadas anteriormente para compreender um tema pré-estabelecido de forma ordenada, obtendo novas informações sobre o assunto abordado (MENDES; SILVEIRA; GALVÃO, 2008).

\section{Informações da amostra}

Por meio do uso deste método, no dia 19 de outubro de 2018, por meio da busca na plataforma CAPES, utilizando da string de busca: "liderança" OR "líder", foram encontrados 2.817 resultados a respeito do tema, sendo restritos a artigos, escritos na língua portuguesa e publicados entre os anos de 2010 a 2018.

Após esta pesquisa, ainda em 19 de outubro de 2018, foi aplicado o segundo filtro na plataforma CAPES, na qual foi utilizado o string de busca: "Liderança feminina" OR "estilos de liderança" OR "competências do líder" OR "o papel do líder" OR "líder coach" OR "motivação e liderança” OR "líder e gestor", localizando um total de 75 artigos, permanecendo com as mesmas restrições.

Com os 75 artigos selecionados foi possível construir uma planilha no Microsoft Excel, utilizando do seu respectivo título, objetivo, metodologia, tema e ano para compor as colunas.

Assim, nos dias 22 e 23 de outubro foi aplicado o terceiro filtro, inicialmente sendo realizada a leitura do título, nas quais foram eliminados 28 artigos por não possuírem relevância para a presente revisão sistemática, levando em consideração a temática exposta mediante o título, visto que eles não haviam relação com o assunto de liderança, apresentando outras temáticas distintas. Também foi retirado 05 artigos por serem de Portugal, tendo como restrição do presente estudo artigos que sejam apenas nacionais, 01 artigo por ser duplicado e 01 artigo por ser do ano de 2009, portanto, não está dentro das restrições para a referente pesquisa, tendo sido determinado apenas estudos entre 2010 a 2018, restando 40 artigos.

Posteriormente, foi realizada uma análise de conteúdo. Na primeira etapa foi aplicada uma pré-análise, ou seja, de forma superficial é observado às primeiras impressões do estudo, a delimitação do que será analisado e a determinação de indicadores. Na segunda etapa é aplicada a exploração do material, a qual é submetido a análise das hipóteses e referencial teórico. Por fim, na terceira etapa é realizada a análise dos resultados e interpretações realizadas (BARDIN, 2011).

Perante tal análise de conteúdo foram eliminados mais 11 artigos, pelo fato de o conteúdo da pesquisa não estar em acordo com a presente revisão sistemática, tendo abordado assuntos e resultados distintos da temática de liderança, restando 29 artigos. Após o uso dos filtros, os 29 artigos selecionados foram agrupados na planilha de acordo com a semelhança dos seus objetivos.

A Figura 1 retrata o protocolo utilizado para a seleção de artigos da revisão sistemática integrativa de forma sintética e cronológica: 
Figura 01 - Processo do protocolo utilizado para a seleção dos artigos

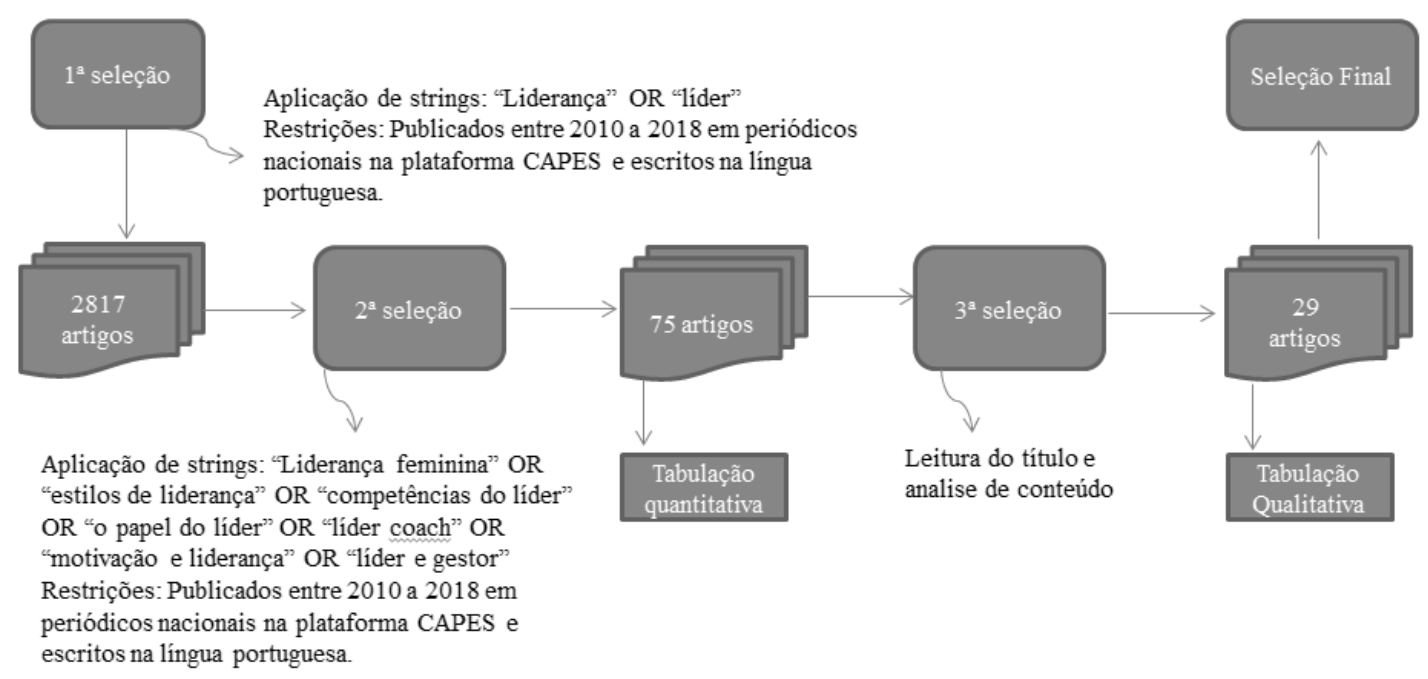

Fonte: Elaborado pelo autor (2018).

\section{Resultados e Discussões}

Para melhor compreender os resultados e discussões deste artigo, esta seção está dividida em: análise quantitativa da revisão sistemática; framework teórico dos artigos selecionados; análise qualitativa dos artigos e discussão.

\section{A trajetória nacional dos estudos sobre liderança organizacional}

De acordo com os dados e informações obtidos por meio da revisão sistemática integrativa, é possível notar que a grande parte dos estudos de liderança publicados em periódicos nacionais entre os anos de 2010 a 2018 encontrados na plataforma CAPES tem como objetivo identificar a visão sobre o estilo de liderança aplicado (12 artigos), seguido por analisar a atuação do líder e os seus resultados (06 artigos), identificar a concepção de liderança (05 artigos), analisar a liderança feminina aplicada (03), identificar o papel do líder (01), analisar a diferença da gestão masculina para a gestão feminina (01) e analisar experiências de coaching (01).

Figura 02 - Objetivos dos estudos

\begin{tabular}{|l|l|} 
& Estilo de liderança aplicado \\
& Atuação do líder e os seus resultados \\
& Identificar a concepção de liderança \\
& Analisar a liderança feminina aplicada \\
& Identificar o papel do líder \\
& Analisar a diferença da gestão masculina para a \\
& gestão feminina \\
& Analisar experiências de coaching \\
&
\end{tabular}

Fonte: Elaborado pelo autor (2018). 
Assim sendo, os principais temas abordados são os estilos de liderança (13), características do líder (06), líder e gestor (03), liderança feminina (04), coaching (01), perfil do líder (02), o papel do líder (01) e o processo de liderança (01), tendo um mesmo artigo abordando um ou mais temas no mesmo estudo.

Dentre estes estudos encontrados com a revisão sistemática, no ano de 2010 retornou 01 artigo, enquanto em 2017 retornaram 05 artigos. Aponta-se uma queda em 2011, que não retornou nenhum artigo, enquanto no ano de 2013 e 2015 retornaram 07 artigos (Figura 03).

Figura 03 - Artigos Encontrados

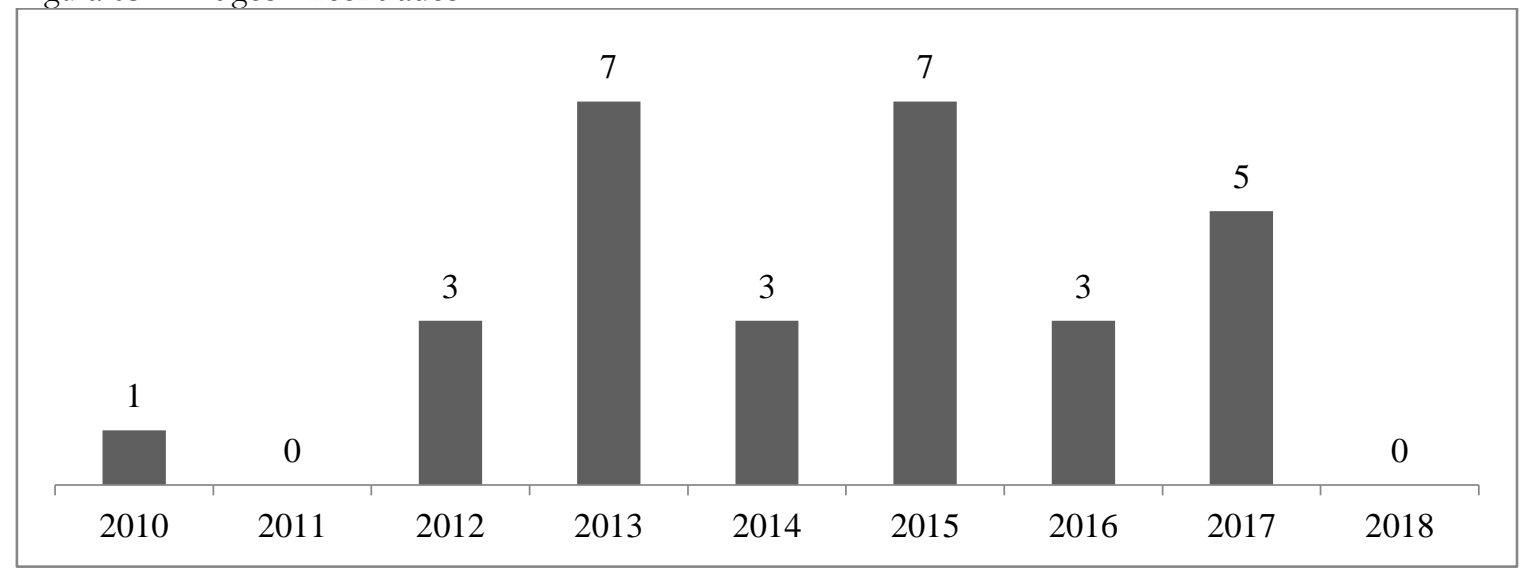

Fonte: Elaborado pelo autor (2018).

No ano de 2010 foi encontrada apenas uma pesquisa sobre estilos de liderança (SILVA, 2010), já no ano de 2012 resultou-se em um artigo de liderança feminina (GOMES et al., 2012), outro em estilos de liderança (SANT'ANNA; PASCHOAL; GOSENDO, 2012) e outro sobre o papel do líder (SILVA FILHO, 2012). Em 2013 foram dois estudos sobre estilos de liderança (FONSECA; PORTO, 2013; LESSA; LUZ; LIMA, 2013), dois sobre o perfil do líder (AGUSTI, 2013; REZENDE et al., 2013), um sobre liderança feminina (NOGUEIRA; KUBO, 2013), outro sobre características do líder (GUERRA; SPIRI, 2013) e um sobre o processo de liderança (OLIVEIRA; DELFINO, 2013).

Já no ano de 2014 foram achados dois a respeito de estilos de liderança (AULER, 2014; SANTOS; MOSCON, 2014) e um sobre características do líder (ASSIS; MAIA, 2014). Em 2015 foram localizados quatro artigos a respeito de estilos de liderança (GONÇALVES et al., 2015; MARQUES; SOUZA; MORI, 2015; NOGUEIRA; COSTA; CLARO, 2015; SCHLEY et al., 2015), três sobre características do líder (GOMES et al., 2015; MARQUES; SOUZA; MORI, 2015; SOUSA, 2015) e duas de liderança feminina (CANABARRO; SALVAGNI, 2015; SOUSA, 2015).

Posteriormente, em 2016 foram duas com a temática de estilos de liderança (BATISTA; KILIMNIK; REIS NETO, 2016; TOMEI; RICHE, 2016) e uma de características do líder (MONTEZELI et al., 2016). Por fim, em 2017 foi localizado três com a temática de líder e gestor (BIANCHI; QUISHIDA; FORONI, 2017; DORNELES; SALVAGNI; NODARI, 2017; SILVA et al., 2017), um sobre coaching (BIRCK; ZILIOTTO, 2017) e um de estilos de liderança (BARBOSA; GAMBI; GEROLAMO, 2017).

\section{Framework teórico da revisão integrativa}

Para melhor compreender os principais avanços dos artigos coletados nessa pesquisa, foi elaborado um framework teórico dividido de forma alfabética e classificado segundo as áreas temáticas: 
Tabela 01 - Síntese dos artigos encontrados na revisão sistemática

\begin{tabular}{|c|c|c|}
\hline Estudos & Síntese & Classificação \\
\hline Assis e Maia (2014) & $\begin{array}{l}\text { O artigo identifica as características pessoas e profissionais } \\
\text { bibliotecários de Goiânia-GO em relação a liderança e as } \\
\text { características da equipe liderada, além de apresentar os desafios } \\
\text { desse profissional em exercer a liderança no ambiente de trabalho. }\end{array}$ & Características do líder \\
\hline Gomes et al. (2015) & $\begin{array}{l}\text { Este trabalho reconhece as percepções dos enfermeiros sobre } \\
\text { liderança e remete à necessidade de repensar a constituinte } \\
\text { liderança na existência profissional }\end{array}$ & Características do líder \\
\hline Guerra e Spiri (2013) & $\begin{array}{l}\text { Este estudo compreende o significado da liderança para o } \\
\text { graduando em enfermagem e a expectativa relacionada à sua } \\
\text { prática profissional }\end{array}$ & Características do líder \\
\hline Montezeli et al. (2016) & $\begin{array}{l}\text { O estudo identifica a concepção de graduandos do último semestre } \\
\text { de um curso de Enfermagem sobre a mobilização da liderança na } \\
\text { prática profissional do enfermeiro }\end{array}$ & Características do líder \\
\hline $\begin{array}{l}\text { Marques, Souza e Mori } \\
\qquad(2015)\end{array}$ & $\begin{array}{l}\text { Este trabalho analisa a atuação do líder no comportamento } \\
\text { organizacional no sistema carcerário de Montes Claros - MG, } \\
\text { identificando os estilos de liderança e o poder de influência do } \\
\text { líder. }\end{array}$ & $\begin{array}{l}\text { Características do líder e } \\
\text { estilos de liderança }\end{array}$ \\
\hline Sousa (2015) & $\begin{array}{l}\text { O artigo analisa diferenças na percepção que os colaboradores } \\
\text { possuem sobre a forma de liderar de gestores homens e mulheres } \\
\text { promovem o bem-estar nas empresas }\end{array}$ & $\begin{array}{l}\text { Características do líder e } \\
\text { liderança feminina }\end{array}$ \\
\hline Birck e Ziliotto (2017) & $\begin{array}{l}\text { O artigo analisa uma experiência do líder coaching realizada com } \\
\text { gestores em contexto escolar, de modo a avaliar as capacidades da } \\
\text { ferramenta neste âmbito. }\end{array}$ & Coaching \\
\hline Auler (2014) & $\begin{array}{l}\text { Identificar os estilos de liderança mais utilizados pelos gerentes } \\
\text { bancários, levando em consideração as variantes de cada agência } \\
\text { pesquisada. }\end{array}$ & Estilos de liderança \\
\hline $\begin{array}{l}\text { Barbosa, Gambi e } \\
\text { Gerolamo (2017) }\end{array}$ & $\begin{array}{l}\text { Este trabalho explora e estabelece relações entre liderança e gestão } \\
\text { da qualidade em empresas brasileiras. }\end{array}$ & Estilos de liderança \\
\hline $\begin{array}{l}\text { Batista, Kilimnik e Reis } \\
\text { Neto (2016) }\end{array}$ & $\begin{array}{l}\text { Os autores identificam qual é a influência do estilo de liderança } \\
\text { sobre a satisfação dos liderados e no desempenho e na } \\
\text { comunicação do líder com o liderado em uma entidade sem fins } \\
\text { econômicos. }\end{array}$ & Estilos de liderança \\
\hline Fonseca e Porto (2013) & $\begin{array}{l}\text { O estudo fornece comprovações de validade de uma escala para } \\
\text { mensurar atitudes perante a liderança transformacional e a } \\
\text { transacional. }\end{array}$ & Estilos de liderança \\
\hline Gonçalves et al. (2015) & $\begin{array}{l}\text { Os autores identificam quais os estilos de liderança e a cultura } \\
\text { organizacional presente em uma instituição de ensino superior e } \\
\text { qual a relação entre eles }\end{array}$ & Estilos de liderança \\
\hline $\begin{array}{l}\text { Lessa, Luz e Lima } \\
\text { (2013) }\end{array}$ & $\begin{array}{l}\text { O artigo investiga os estilos de liderança dos professores- } \\
\text { treinadores através da conduta percebida por alunos-atletas, além } \\
\text { de identificar o perfil de liderança predominante dos professores- } \\
\text { treinadores da atualidade de Teresina-PI. }\end{array}$ & Estilos de liderança \\
\hline $\begin{array}{c}\text { Nogueira, Costa e Claro } \\
\text { (2015) }\end{array}$ & $\begin{array}{l}\text { O artigo analisa a relação entre os estilos de liderança e o } \\
\text { comprometimento organizacional afetivo em trabalhadores de } \\
\text { uma empresa privada em São Paulo }\end{array}$ & Estilos de liderança \\
\hline $\begin{array}{l}\text { Sant'Anna, Paschoal e } \\
\text { Gosendo (2012) }\end{array}$ & $\begin{array}{l}\text { Os autores testam a relação do estilo de liderança com o bem-estar } \\
\text { no trabalho }\end{array}$ & Estilos de liderança \\
\hline Santos e Moscon (2014) & 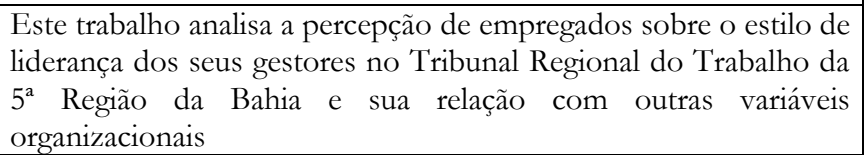 & Estilos de liderança \\
\hline Schley et al. (2015) & $\begin{array}{l}\text { O artigo identifica a percepção dos líderes em relação ao estilo de } \\
\text { liderança adotado aos seus subordinados em uma das lojas de uma } \\
\text { rede de supermercados da mesorregião central do Paraná }\end{array}$ & Estilos de liderança \\
\hline Silva (2010) & $\begin{array}{l}\text { O estudo analisa os tipos de lideranças ideais para o Hospital } \\
\text { Regional de Presidente Prudente - SP }\end{array}$ & Estilos de liderança \\
\hline Tomei e Riche (2016) & $\begin{array}{l}\text { Os autores analisam estilos de liderança e o desempenho } \\
\text { organizacional a partir da comparação de duas empresas familiares } \\
\text { brasileiras de pequeno porte do comércio varejista }\end{array}$ & Estilos de liderança \\
\hline $\begin{array}{l}\text { Bianchi, Quishida e } \\
\text { Foroni (2017) }\end{array}$ & $\begin{array}{l}\text { O estudo analisa a atuação do líder como elo fundamental na } \\
\text { gestão estratégica de pessoas por meio de reflexões com base no } \\
\text { referencial teórico }\end{array}$ & Líder e gestor \\
\hline
\end{tabular}




\begin{tabular}{|c|l|c|c|}
$\begin{array}{c}\text { Dorneles, Salvagni e } \\
\text { Nodari (2017) }\end{array}$ & $\begin{array}{l}\text { Os autores identificam como a prática da liderança pode modificar } \\
\text { e melhorar o relacionamento entre os colaboradores e seus } \\
\text { gestores em uma organização }\end{array}$ & Líder e gestor \\
\hline Silva et al. (2017) & $\begin{array}{l}\text { Os autores destacam a relevância da aplicação de conceitos e } \\
\text { práticas envolvendo processos de liderança no ambiente bancário }\end{array}$ & Líder e gestor \\
\hline $\begin{array}{c}\text { Canabarro e Salvagni } \\
(2015)\end{array}$ & $\begin{array}{l}\text { Este trabalho analisa a trajetória de lideranças femininas nas suas } \\
\text { devidas organizações, mostrando os desafios encontrados pela } \\
\text { liderança feminina. }\end{array}$ & Liderança Feminina \\
\hline Gomes et al. (2012) & $\begin{array}{l}\text { O artigo analisa as representações da mídia sobre as estratégias de } \\
\text { liderança feminina da presidente do Clube de Regatas Flamengo } \\
\text { (CRF) }\end{array}$ & Liderança Feminina \\
\hline Nogueira e Kubo (2013) & $\begin{array}{l}\text { Os autores analisam os sentidos de liderança por mulheres } \\
\text { executivas brasileiras e os seus elementos dificultadores }\end{array}$ & Liderança Feminina \\
\hline Silva Filho (2012) & $\begin{array}{l}\text { O trabalho examina aspectos humanos que influenciam a liderança } \\
\text { num ambiente corporativo }\end{array}$ & Papel do líder \\
\hline Agusti (2013) & $\begin{array}{l}\text { O autor do artigo estabelece um perfil básico para o líder do setor } \\
\text { de manutenção das empresas }\end{array}$ & Perfil do líder \\
\hline Rezende et al. (2013) & $\begin{array}{l}\text { Este estudo reconhece as dificuldades que os enfermeiros } \\
\text { enfrentam para pôr em prática o exercício da liderança na } \\
\text { enfermagem }\end{array}$ & Perfil do líder \\
\hline $\begin{array}{c}\text { Oliveira e Delfino } \\
\text { (2013) }\end{array}$ & $\begin{array}{l}\text { Os autores identificam como está caracterizada a produção } \\
\text { científica sobre o processo de liderança no período de 1981 a 2010 }\end{array}$ & Processo de liderança \\
\hline
\end{tabular}

Fonte: Elaborado pelo autor (2018).

\section{Análise qualitativa da produção nacional pós-2010}

Por meio da análise de conteúdo de Bardin (2011), foram selecionados os estudos que estavam diretamente relacionados à abordagem provocada pela presente revisão sistemática, sendo liderança feminina, estilos de liderança, perfil do líder, competências do líder, coaching, líder e gestor e motivação e liderança.

Assim, é possível notar que as pesquisas de liderança voltadas às características e o perfil do líder dos anos de 2010 a 2018 estão focadas no estudo da percepção e análise da liderança dentro da área da saúde, buscando relacionar os dois campos para alcançar resultados mais efetivos dentro das clinicas e hospitais por meio dos médicos e enfermeiros chefe (MONTEZELI et al., 2016; GOMES et al., 2015; GUERRA; SPIRI, 2013; REZENDE et al., 2013).

O estudo de Guerra e Spiri (2013) e Montezeli et al. (2016) abordam as perspectivas que os estudantes de graduação da área da saúde tem quanto a liderança em seu meio profissional em formação, enquanto Gomes et al. (2015) já remete em sua pesquisa a forma como os enfermeiros veem a necessidade da abordagem de tal questão em seu meio profissional. Também, Rezende et al. (2013) busca analisar as dificuldades dos enfermeiros em colocar em pratica o exercício da liderança, mostrando que tal abordagem tem sido um desafio pela falta de competência para exercer tal papel, sendo necessário a instigação de tal função durante a graduação.

Esses estudos demonstram a necessidade do ensino e aplicação da liderança durante a formação profissional não apenas em cursos de gestão, mas em todas as áreas, pois outros profissionais também exercem cargos de gestão dentro de suas atividades. Entretanto, quando tal situação ocorre, de acordo com os estudos apresentados, eles se demonstram despreparados para assumir tais funções de um líder, em especial por não terem contato com tais teorias e aplicabilidades em sua formação.

Já dentre os estudos selecionados a respeito de estilos de liderança demonstram que o foco principal dos estudos são os estilos modernos de liderar, como o transformacional, transacional, situacional e relacional (BARBOSA; GAMBI; GEROLAMO, 2017; BATISTA; KILIMNIK; REIS NETO, 2016; FONSECA; PORTO, 2013; GONÇALVES et al., 2015; NOGUEIRA; COSTA; CLARO, 2015; SANT'ANNA; PASCHOAL; GOSENDO, 2012; SANTOS; MOSCON, 2014; TOMEI; RICHE, 2016). 
Esses estudos de estilos de liderança analisados tem o intuito de aprimorar a gestão da qualidade da organização junto da liderança (BARBOSA; GAMBI; GEROLAMO, 2017) e a satisfação e o bem-estar dos liderados (BATISTA; KILIMNIK; REIS NETO, 2016; SANT'ANNA; PASCHOAL; GOSENDO, 2012), assim como demonstram a existência da relação entre o estilo de liderança e a cultura organizacional (GONÇALVES et al., 2015) e também com o comprometimento e o desempenho organizacional (NOGUEIRA; COSTA; CLARO, 2015; SANTOS; MOSCON, 2014; TOMEI; RICHE, 2016). Dessa forma, esses estudos de estilos de liderança vêm com a intuito de demonstrar quais as qualificações que a aderência de determinado estilo de liderança pode trazer para a organização. Por fim, Fonseca e Porto (2013) propõe a validação de uma escala para a análise da liderança transformacional e transacional.

Outrossim, relacionado ao empoderamento feminino, aparece nas pesquisas em meio ao ambiente organizacional, sendo expostos por meio de estudos visando analisar a representatividade da mulher como líderes no círculo organizacional, avaliando a gestão e trajetória das executivas para chegarem aos seus postos atuais (CANABARRO; SALVAGNI, 2015; SOUSA, 2015; GOMES et al., 2012; NOGUEIRA; KUBO, 2013).

Esses quatro estudos realizam um estudo de caso com gestoras brasileiras como líderes em suas organizações, mostrando as dificuldades enfrentadas por elas, tendo como principais empecilhos a maturidade e o comprometimento da equipe, juntamente do machismo (NOGUEIRA; KUBO, 2013). Outra dificuldade explorada é o conflito do equilíbrio entre a vida pessoal, possuindo casa e filhos para cuidar, com a vida profissional (CANABARRO; SALVAGNI, 2015). Gomes et al. (2012) exorta a forma como a gestora do Clube de Regatas Flamengo gerenciou o clube até então, apontando as suas características pessoais, sua postura e as suas adversidades para assumir tal posição.

\section{Discussão}

Conforme apontado na introdução por Yamafuko e Silva (2015), os estudos encontrados com a revisão sistemática integrativa vêm para reforçarem que não existe uma definição exata de liderança, ao passo de que todas as pesquisas de perfil e características do líder encontradas no estudo trouxeram novos conceitos, questões e abordagens a serem levadas em consideração para o mundo contemporâneo (AGUSTI, 2013; ASSIS; MAIA, 2014; GOMES et al., 2015; GUERRA; SPIRI, 2013; MARQUES; SOUZA; MORI, 2015; MONTEZELI et al.; 2016; OLIVEIRA; DELFINO, 2013; REZENDE et al., 2013; SILVA FILHO, 2012; SOUSA, 2015).

A análise quantitativa da presente revisão sistemática apresentada mostra uma grande quantidade de estudos sobre estilos de liderança (41\%) em comparação aos outros estudos tendo outras linhas como foco, como já apontado por Fonseca, Porto e Borges-Andrade (2015). Enquanto a gestão feminina (4\%) e coaching $(3 \%)$ ainda possuem muito potencial de exploração, levando em consideração que são fenômenos em pauta na sociedade contemporânea (NORO; STÜKER; OLIVEIRA, 2015; ROCHACOUTINHO; COUTINHO, 2011).

Foi possível notar que não existe uma constância na publicação de estudos de liderança com o passar dos

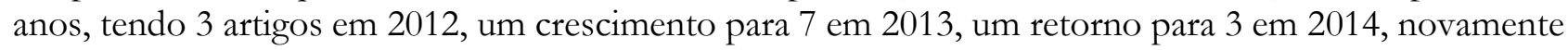
7 em 2015, 3 em 2016 e por fim 5 em 2017, nenhuma publicação em 2018 até 19 de outubro, quando se deu início a presente revisão sistemática integrativa (FONSECA; PORTO; BORGES-ANDRADE, 2015).

Na temática de estilos de liderança, é claro que os mais apontados são o estilo transformacional e o transacional, sendo encontrados em 06 estudos diferentes, que estão tomando espaço nas organizações e mudando o jeito de liderar para uma forma mais efetiva do que a tradicional (BARBOSA; GAMBI; 
GEROLAMO, 2017; BATISTA; KILIMNIK; REIS NETO, 2016; FONSECA; PORTO, 2013; GONÇALVES et al., 2015; SANTOS; MOSCON, 2014; TOMEI; RICHE, 2016).

Outrossim está em que alguns autores usam nomes diferentes para os mesmos conceitos de estilos de liderança e trazendo algumas novidades, como Schley et al. (2015), que ao invés de usar os nomes tradicionais, como autocrático, democrático, carismático e transformacional, utilizou-se de coercitivo, dirigente, modelador, afetivo, democrático e treinador.

O Framework construído permite uma comparação clara e coesa dos estudos publicados no decorrer dos anos com base na temática e no conteúdo abordado em cada pesquisa, demonstrando de uma forma prática a síntese do que tem sido estudo na área de liderança nos últimos anos.

É possível perceber ainda que é necessário distinguir a diferença entre gestor e líder, visualizando todas as possibilidades positivas que tal diferenciação traz a organização, sendo necessário para o seu desenvolvimento de forma efetiva (BIANCHI; QUISHIDA; FORONI, 2017; DORNELES; SALVAGNI; NODARI, 2017; SILVA et al., 2017), corroborando com Bertolla et al. (2014).

Por fim, em relação aos temas coaching e liderança feminina, foram encontrados apenas 04 artigos sobre liderança feminina (CANABARRO; SALVAGNI, 2015; SOUSA, 2015; GOMES et al., 2012; NOGUEIRA; KUBO, 2013) e 01 sobre coaching (BIRCK; ZILIOTTO, 2017), não retratando o mesmo movimento ocorrido nos estudos em livros para o mercado (NORO; STÜKER; OLIVEIRA, 2015; ROCHA-COUTINHO; COUTINHO, 2011).

\section{Considerações Finais}

O presente estudo atingiu o seu objetivo geral e objetivos específicos ao analisar de forma integrativa e sistêmica os trabalhos de liderança publicados em periódicos nacionais na plataforma da CAPES de 2010 a 2018, de forma a beneficiar com informações a todos os líderes, organizações, pesquisadores e estudiosos.

Também foi exposto no transcorrer das seções um embasamento teórico sobre a temática de liderança, por meio de uma análise dos estudos nacionais de liderança publicados de 2010 a 2018 na plataforma CAPES de forma sistemática e integrativa; e expondo a comparação dos estudos analisados de forma ordenada, orientando os estudos a serem desenvolvidos na área.

Tendo em vista as teorias levantadas e as informações apresentadas por meio da revisão sistemática integrativa a respeito dos estudos de liderança publicados em periódicos nacionais na plataforma CAPES entre 2010 a 2018, pode-se concluir que os temas mais abordados são a respeito dos estilos de liderança, com ênfase para o estilo transformacional e transacional. Já temas que estão em grande expansão no mercado, como coaching e a liderança feminina, ainda são pouco encontrados na área acadêmica.

Dessa forma, o presente estudo contribui de forma clara para o desenvolvimento da pesquisa e da ciência nacional na área de liderança, permitindo um panorama geral e crítico do que vem sendo publicado em periódicos nacionais nos últimos anos para que se possa observar as possíveis falhas, as temáticas em deficiência de exploração e, dessa forma, dar continuidade as temáticas desses estudos, bem como a identificação de novas lacunas de pesquisa com o objetivo de alavancar a pesquisa nacional.

Como limitações deste estudo é importante ressaltar que as dificuldades encontradas para a elaboração do estudo remetem ao fato de existirem poucas revisões sistemáticas na área de administração e liderança, não havendo um padrão específico ou um caminho a ser seguido. Além disso, o estudo limitou-se a 
focalizar em estudos nacionais e utilizando da plataforma CAPES, com strings restritos e excludentes, de forma a abrir espaço para utilização de outras bases de dados internacionais, como Web of Science e Scopus.

Ao final, como forma de propor uma agenda de pesquisa futura, propõe-se:

1) Pesquisas de revisão sistemática integrativa de forma mais restritiva e internacional dentro da temática de liderança, como estilos de liderança, liderança feminina ou qualquer outro tema, se aprofundando na área e buscando maior fidelidade aos resultados.

2) A exploração de temas mais contemporâneos, como a liderança feminina e o coaching, podendo realizar investigações a respeito dos desafios para aplicações de tais abordagens e como alcançar estratégias mais efetivas para superá-las.

3) A padronização dos termos relacionados a área de liderança, buscando a facilidade do estudo e aplicação da teoria para com a prática, fugindo do que acontece com os estilos de liderança, por exemplo, onde existem diversos termos para retratar o mesmo assunto, como mostrado na represente revisão sistemática integrativa.

\section{Referências}

AARONS, G. A. et al. The Roles of System and Organizational Leadership in System-Wide EvidenceBased Intervention Sustainment: A Mixed-Method Study. Administration and Policy in Mental Health and Mental Health Services Research, 43(6), 991-1008, 2016.

AGUSTI, A. L. Líder da manutenção: proposição de perfil com base em um estudo biblioFigura. GEPROS. Gestão da Produção, Operações e Sistemas, vol. 8, nº 4, p. 77-91, 2013.

ARNOLD, K. A. et al. Leadership styles, emotion regulation, and burnout. Journal of Occupational Health Psychology, 20(4), 481-490, 2015.

ARRUDA, Â. M. F.; CHRISÓSTOMO, E.; RIOS, S. S. A importância da liderança nas organizações. Revista Razão Contábil \& Finanças, v. 1, n. 1, p. 1-15, 2010.

ASSIS, C. C.; MAIA, L. C. G. Análise do Perfil de Liderança do Bibliotecário Gestor na Cidade de Goiânia - Go. Informação \& Informação, vol. 19, n. 1, p. 185-205, 2014.

AULER, D. Os estilos de liderança na gestão dos serviços bancários. Revista de Carreiras e Pessoas, vol. 4, n. 2, 2014.

BARBOSA, F. M.; GAMBI, L. N.; GEROLAMO, M. C. Liderança e gestão da qualidade - um estudo correlacional entre estilos de liderança e princípios da gestão da qualidade. Gestão \& Produção, vol. 24, n. 3, p. 438-449, 2017.

BARDIN, L. Análise de conteúdo. São Paulo: Edições 70. 2011.

BATISTA, N. P. O.; KILIMNIK, Z. M.; REIS NETO, M. T. Influência dos estilos de liderança na satisfação no trabalho: um estudo em uma entidade de fins não econômicos. Revista de Gestão e Tecnologia, vol. 6, n. 3, 2016.

BEDI, A.; ALPASLAN, C. M.; GREEN, S. A Meta-analytic Review of Ethical Leadership Outcomes and Moderators. Journal of Business Ethics, 139(3), 517-536, 2015.

BEHNKE, M. T. Gestão de Pessoas: Artigos reunidos. 1. ed. Curitiba: InterSaberes, 2014.

BERG, M. E.; KARLSEN, J. T. A study of coaching leadership style practice in projects. Management Research Review, 39(9), 1122-1142, 2016. 
BERGAMINI, C. W. Liderança: Administração do Sentido. 2. ed. São Paulo: Atlas S.A., 2009.

BERTOLLA, A. et al. O Gestor Como Líder no Processo de Desenvolvimento de Pessoas. $4^{\text {a }}$ Semana Internacional de Engenharia e Economia FAHOR, 2014.

BIANCHI, E. M. P. G.; QUISHIDA, A.; FORONI, P. G. Atuação do líder na gestão estratégica de pessoas: reflexões, lacunas e oportunidades. Revista de Administração Contemporânea, vol. 21, n. 1, p. 41-61, 2017.

BIRCK, F. K.; ZILIOTTO, D. M. Coaching na educação? Pesquisa-ação com gestores escolares. Acta Scientiarum Human and Social Sciences, vol. 39, n. 2, 2017.

BOTELHO, J. C.; KROM, V. Os estilos de Liderança nas Organizações. Anais - XIV Encontro Latino Americano de Iniciação Científica e X Encontro Latino Americano de Pós-Graduação. Universidade do Vale do Paraíba, 2010.

CANABARRO, J. R. S.; SALVAGNI, J. Mulheres líderes: as desigualdades de gênero, carreira e família nas organizações de trabalho. Revista de Gestão e Secretariado, vol. 6, n. 2, 2015.

CARSON, J. B.; TESLUK, P. E.; MARRONE, J. A. Shared leadership in teams: An investigation of antecedent conditions and performance. Academy of Management Journal, vol. 50, p. 1217-1234, 2007.

CARVALHO, M. S.; PALMEIRA, E. M.; MARIANO, M. G. H. Liderança baseada na motivação e desenvolvimento de pessoal como estratégia de competitividade das organizações. Observatório de la Economia Latino-americano, n. 167, 2012.

CHEN, G. et al. A multilevel study of leadership, empowerment, and performance in teams. Journal of Applied Psychology, vol. 92, p. 331-346, 2007.

D'INNOCENZO, L.; MATHIEU, J. E.; KUKENBERGER, M. R. A Meta-Analysis of Different Forms of Shared Leadership-Team Performance Relations. Journal of Management, 42(7), 1964-1991, 2016.

DORNELES, E. L. M.; SALVAGNI, J.; NODARI, C. H. A liderança como diferencial nas organizações: Um estudo sobre a percepção dos gestores. Holos, vol. 8, p. 172-190, 2017.

DRUSKAT, V. U.; WHEELER, J. V. Managing from the boundary: The effective leadership of selfmanaging work teams. Academy of Management Journal, vol. 46, p. 435-457, 2003.

EISENBEISS, S. A.; VAN KNIPPENBERG, D.; BOERNER, S. Transformational leadership and team innovation: Integrating team climate principles. Journal of Applied Psychology, vol. 93, p. 1438-1446, 2008.

EPITROPAKI, O. et al. Leadership and followership identity processes: A multilevel review. The Leadership Quarterly, 28(1), 104-129, 2017.

FONSECA, A. M. O.; PORTO, J. B. Validação fatorial de escala de atitudes frente a estilos de liderança. Avaliação Psicológica, vol. 12, n. 2, 2013.

GOMES, E. et al. As Representações da Mídia sobre a Gestão Feminina no Clube de Regatas Flamengo. PODIUM Sport, Leisure and Tourism Review, vol. 1, n. 1, 2012.

GOMES, L. A. et al. Percepções de enfermeiros sobre liderança: um repensar necessário. Revista Eletrônica Gestão \& Saúde, vol. 6, n. 3, pp. 2711-2722, 2015.

GONÇALVES, A. et al. A relação entre liderança e cultura organizacional: um estudo realizado em uma IES. Gestão da Produção, Operações e Sistemas, vol. 10, n. 2, pp. 85-100, 2015.

GREENHALGH, T. Papers that summarize other papers (systematic review and meta-analyses). British Medical Journal, London, v. 315, n. 7109, p. 672-675, Sep. 1997. 
GUERRA, K. J.; SPIRI, W. C. Compreendendo o significado da liderança para o aluno de graduação em enfermagem: uma abordagem fenomenológica. Revista Brasileira de Enfermagem, vol. 66, n. 3, pp. 399-405, 2013.

HILLER, N. J.; DAY, D. V.; VANCE, R. J. Collective enactment of leadership roles and team effectiveness: A field study. Leadership Quarterly, vol. 17, p. 387-397, 2006.

IVAN, M. V.; TERRA, L. A. A. A influência da liderança na dinâmica organizacional. Caderno Profissional de Administração - UNIMEP, 2017, vol. 07, n. 01.

LESSA, L. F.; LUZ, E. W. M.; LIMA, S. F. Percepção dos alunos-atletas sobre o estilo de liderança dos professores-treinadores de Teresina-PI. Revista Brasileira de Futsal e Futebol, v. 5, n. 18, 2013.

LIMA, T. C. S.; MIOTO, R. C. T. Procedimento metodológico na construção do conhecimento científico: A pesquisa bibliográfica. Katálysis, 2007, vol. 10, pp. 37-45.

MARQUES, M. L. O.; SOUZA, D. F. F.; MORI, A. L. P. Liderança e comportamento organizacional: um estudo de caso no sistema carcerário. Revista da Universidade Vale do Rio Verde, vol. 13, n. 1, 2015.

MASCARENHAS, S. A. Metodologia científica. São Paulo: Pearson Education Brasil, 2012.

MENDES, K. D. S.; SILVEIRA, R. C. C. P.; GALVÃO, C. M. Revisão integrativa: método de pesquisa para a incorporação de evidências na saúde e na enfermagem. Texto Contexto Enfermagem, Florianópolis, v. 17, n. 4, p. 758-764, 2008.

MONTEZELI, J. H. et al. Mobilização da liderança: concepção de graduandos em enfermagem. Revista de Pesquisa: Cuidado é Fundamental, vol. 8, n. 4, p. 5114-5119, 2016.

MORGESON, F. P. The external leadership of self-managing teams: Intervening in the context of novel and disruptive events. Journal of Applied Psychology, vol. 90, p. 497-508, 2005.

MORGESON, F. P.; DERUE, D. S. Event criticality, urgency, and duration: Understanding how events disrupt teams and influence team leader intervention. Leadership Quarterly, vol. 17, p. 271-287, 2006.

MORGESON, F. P.; DERUE, D. S.; KARAM, E. P. Leadership in Teams: A Functional Approach to Understanding Leadership Structures and Processes. Journal of Management, vol. 36, p. 5-39, 2010.

NOGUEIRA, E. C. O. R.; KUBO, E. K. M. Sentidos do exercício da liderança por mulheres executivas brasileiras. Revista de Gestão e Secretariado, vol. 4, n. 2, pp. 114-134, 2013.

NOGUEIRA, M. L. O.; COSTA, L. V.; CLARO, J. A. C. S. Relação entre estilo de liderança e comprometimento organizacional afetivo. Revista de Administração, Contabilidade e Economia RACE, vol. 14, n. 2, 2015.

NORO, G. B.; STÜKER, C.; OLIVEIRA, J. H. R. A relação das características inerentes aos perfis de liderança no desenvolvimento do processo coaching. Revista Capital Cientifico, 2015, vol. 13, n. 01.

PEARCE, C. L.; SIMS, H. P. Vertical versus shared leadership as predictors of the effectiveness of change management teams: An examination of aversive, directive, transactional, transformational, and empowering leader behaviors. Group Dynamics: Theory, Research, and Practice, vol. 6, p. 172-197, 2002.

OLIVEIRA, F. P.; DELFINO, I. A. L. Produção Científica sobre o Processo de Liderança. Revista de Administração, Ciências Contábeis e Sustentabilidade, vol. 3, n. 4, p. 104-126, 2013.

REZENDE, B. C. et al. Dificuldades enfrentadas pelos enfermeiros na prática da liderança em enfermagem: uma revisão da literatura. Revista Eletrônica Gestão e Saúde, vol. 4, n. 2, pp. 2273-2288, 2013. 
ROCHA-COUTINHO, M. L.; COUTINHO, R. R. Mulheres brasileiras em posições de liderança: Novas perspectivas para antigos desafios. Economia Global e Gestão, 2011, vol. 16, n. 01, pp. 61-79.

SANT'ANNA, L. L.; PASCHOAL, T.; GOSENDO, E. E. M. Bem-estar no Trabalho: Relações com Estilos de Liderança e Suporte para Ascensão, Promoção e Salários. Revista de Administração Contemporânea, vol. 16, n. 5, pp. 744-764, 2012.

SANTOS, M. D. M.; MOSCON, D. C. B. Modelos de liderança e percepção do trabalhador sobre a organização empregadora: Um estudo no TRT-BA. Seminário Estudantil de Produção Acadêmica, vol. 13, 2014.

SCHAUBROECK, J.; LAM, S. S. K.; CHA, S. E. Embracing transformational leadership: Team values and the impact of leader behavior on team performance. Journal of Applied Psychology, vol. 92, p. 1020-1030, 2007.

SCHLEY, J. et al. Estilos de liderança: um estudo sobre a percepção dos funcionários de um supermercado da mesorregião central do Paraná. Revista de Administração IMED, vol. 5, n. 2, 2015.

SIDANI, Y. M.; KONRAD, A.; KARAM, C. M. From female leadership advantage to female leadership deficit: A developing country perspective. The Career Development International, 20(3), 273-292, 2015.

SILVA, F. A. et al. Análise da atuação do gerente bancário como líder de equipe em agências do interior paulista. Revista Fatec Zona Sul - REFAS, vol. 3, n. 4, 2017.

SILVA, J. J. O papel do líder na gestão de conflitos e mudança da cultura organizacional dentro de um ambiente hospitalar. Colloquium Humanarum, vol. 7, n. 2, pp. 01-06, 2010

SILVA FILHO, A. M. Criatividade no Ambiente Corporativo - O Papel do Líder. Revista Espaço Acadêmico, n. 134, 2012.

SOUSA, F. C. Percepção de Diferenças Atribuíveis ao Gênero dos Líderes na Promoção do Bem-Estar nas Empresas. Teoria e Prática em Administração, vol. 5, n. 1, p. 105-129, 2015.

TOMEI, P. A.; RICHE, L. Estilo de liderança e desempenho organizacional: uma descrição comparativa de duas empresas. Contabilidade, Gestão e Governança, vol. 19, n. 1, p. 108-125, 2016.

WAGEMAN, R. How leaders foster self-managing team effectiveness: Design choices versus hands-on coaching. Organization Science, vol. 12, p. 559-577, 2001.

WOLFF, L.; CABRAL, P. M. F.; LOURENÇO, P. R. M. R. S. O Papel da Liderança na Eficácia das Equipes de Trabalho. Revista Gestão \& Tecnologia, 2013, vol. 13, n. 01, pp. 177-204.

YAHAYA, R.; EBRAHIM, F. Leadership styles and organizational commitment: literature review. Journal of Management Development, 35(2), 190-216, 2016.

YAMAFUKO, E. L.; SILVA, J. J. Liderança nas Organizações. Colloquium Humanarum, 2015, vol. 12, pp. 86-93. 